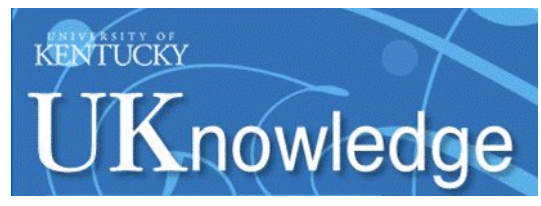

University of Kentucky

UKnowledge

9-1-2014

\title{
Recent Ideas on the Calculation of Lepton Anomalous Magnetic Moments
}

\author{
Michael I. Eides \\ University of Kentucky, eides@pa.uky.edu
}

Follow this and additional works at: https://uknowledge.uky.edu/physastron_facpub

Part of the Astrophysics and Astronomy Commons, and the Physics Commons

Right click to open a feedback form in a new tab to let us know how this document benefits you.

\section{Repository Citation}

Eides, Michael I., "Recent Ideas on the Calculation of Lepton Anomalous Magnetic Moments" (2014).

Physics and Astronomy Faculty Publications. 312.

https://uknowledge.uky.edu/physastron_facpub/312

This Article is brought to you for free and open access by the Physics and Astronomy at UKnowledge. It has been accepted for inclusion in Physics and Astronomy Faculty Publications by an authorized administrator of UKnowledge. For more information, please contact UKnowledge@lsv.uky.edu. 


\section{Recent Ideas on the Calculation of Lepton Anomalous Magnetic Moments}

Digital Object Identifier (DOI)

http://dx.doi.org/10.1103/PhysRevD.90.057301

Notes/Citation Information

Published in Physical Review D: Particles, Fields, Gravitation, and Cosmology, v. 90, no. 5, article 057301, p. 1-4.

@2015 American Physical Society

The copyright holder has granted permission for posting the article here. 


\title{
Recent ideas on the calculation of lepton anomalous magnetic moments
}

\author{
Michael I. Eides* \\ Department of Physics and Astronomy, University of Kentucky, Lexington, Kentucky 40506, USA
}

(Received 2 March 2014; published 16 September 2014)

\begin{abstract}
We discuss the recent claim [G. Mishima, arXiv:1311.7109; M. Fael and M. Passera, arXiv:1402.1575 [Phys. Rev. D (to be published)]] about discovery of a nonperturbative quantum-electrodynamic contribution of order $(\alpha / \pi)^{5}$ to lepton anomalous magnetic moments. We explain why this nonperturbative correction does not exist.
\end{abstract}

Calculation of the QED corrections to lepton anomalous magnetic moments is the classical playground of perturbation theory. In fact, the very first prediction of the newly minted QED was the Schwinger's calculation of the oneloop correction to the electron anomalous magnetic moment (AMM) [1]. Nowadays all QED corrections to the electron AMM up to and including the five-loop $(\alpha / \pi)^{5}$ contributions are calculated; see the review [2].

It was claimed recently $[3,4]$ that the perturbative calculation of the QED contributions to lepton AMMs is incomplete and should be amended by accounting for a "nonperturbative" effect connected with the positronium poles in the photon polarization operator. According to $[3,4]$, these positronium poles in the exact polarization operator generate a new contribution of order $(\alpha / \pi)^{5}$ that is missed in the standard perturbative treatment and that should be added to the perturbative one. If correct, the conclusion about the missing positronium poles would affect many other high-order QED calculations.

The problem of accounting for the positronium poles in the polarization operator in low energy QED calculations was addressed, exhaustively explored, and solved a long time ago in [5] (see also [6]). It was proved that in almost all low energy perturbative calculations, when the momentum $k$ flowing through the polarization operator is far from the positronium singularities $\left|k^{2}-4 m^{2}\right|>\alpha^{2} m^{2}$, perturbative contributions provide a complete QED result. Inclusion of positronium poles in this situation means an ill-defined double counting. The only exception to this rule is the case when the polarization operator enters the diagram in the vicinity of the positronium poles $\left|k^{2}-4 m^{2}\right| \leq \alpha^{2} m^{2}$. Then accounting for the positronium singularities becomes mandatory. An example of such a situation is provided by the vacuum polarization insertion in the diagram with the virtual one-photon annihilation of positronium. The contribution of the positronium singularities to the positronium hyperfine splitting in this case was calculated in [6].

\footnotetext{
*Also at the Petersburg Nuclear Physics Institute, Gatchina, St. Petersburg 188300, Russia. eides@pa.uky.edu; eides@thd.pnpi.spb.ru
}

Below we will consider in more detail calculation of the polarization operator contribution in Fig. 1 and demonstrate that the standard perturbative contributions give the complete QED contribution to AMM. Nothing we will say below is new. It is all contained in $[5,6]$, but the claims made in $[3,4]$ show that the old arguments deserve to be repeated.

We start with a qualitative discussion. Recall how the formation of a QED bound state is described in the diagrammatic language. Consider an electron-positron four-point Green function (the polarization operator is just a special case of this four-point function). This Green function has a series of poles in the $s$ channel $\left(s=k^{2}\right)$ corresponding to the energy levels of the electron-positron bound states-levels of positronium of the form $Z_{n}(\alpha) /\left(s-E_{n}^{2}\right), E_{n}=2 m-\alpha^{2} m /\left(4 n^{2}\right)$. These poles can be considered as singularities (poles) in $\alpha$, and an expansion in powers of $\alpha$ is clearly illegitimate in the vicinity of the poles, when $\left|s-4 m^{2}\right| \leq m^{2} \alpha^{2}$. Besides bound states the singularities in $\alpha$ are due also to the continuum scattering states near the threshold, $s-4 m^{2} \leq m^{2} \alpha^{2}$; see [5,6]. It is well known that these singularities arise as a result of summation of an infinite series of the perturbation theory diagrams; see, e.g., [7]. Consider the diagrams with the (Coulomb) photon exchanges between the electron and positron lines that are responsible for the formation of the bound state. For the generic external momenta each extra exchanged photon carries an extra suppression factor $\alpha \sim 1 / 137$, and the diagrams with a large number of photon exchanges are strongly suppressed. How is it possible that the sum of these diagrams develops poles at $s \sim 4 m^{2}$ corresponding to the positronium bound states? The only way it can happen is if the series diverges at $s \sim 4 m^{2}$ and all terms in the series are of the same order in

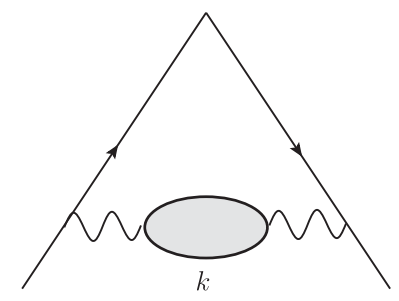

FIG. 1. Polarization operator contribution to AMM. 
the vicinity of $s \sim 4 m^{2}$. At first glance this cannot happen since, as we just explained, addition of an extra rung to the ladder of exchanged photons results in an extra small factor $\alpha$. But not so fast-this simpleminded consideration completely ignores the kinematic dependence of the graphs. A simple calculation shows that at $s \sim 4 m^{2}$ each extra photon is accompanied by an extra factor $1 / v \sim 1 / \alpha$ $\left(v=\sqrt{1-4 m^{2} / s}\right)$. As a result all diagrams are of the same order and the series diverges at $s \sim 4 m^{2}$. We can easily sum the infinite series of diagrams with the Coulomb exchanges far from the threshold, and check that the sum really develops poles at the positions of the positronium energy levels. For our goals it is important to emphasize that the positronium poles in the full polarization operator arise as a result of summation of an infinite series of one-particle irreducible perturbation theory diagrams, and these poles arise at the specific values of the four-momentum flowing through these perturbation theory diagrams.

Now we are ready to return to the problem of accounting for many Coulomb exchanges inside the polarization operator in the diagram in Fig. 1. Let us consider the contribution to AMM provided by the diagram in Fig. 1, where the blob is substituted by any finite-order perturbation theory diagram for the polarization operator. It is well known that the structure of singularities in the complex $k_{0}$ plane is such that it allows the Wick rotation; see, e.g., $[7,8]$. After the Wick rotation, the loop (and polarization operator) momentum is spacelike, $k^{2}=-k_{E}^{2}<0$. Simply from dimensional considerations we know that the characteristic integration loop momentum in this diagram is determined by the electron mass, and the dominant contribution to the diagram is produced by the region of integration momenta where Euclidean momenta $k_{E}^{2} \leq m^{2}$. This is true for calculation of the leading Schwinger contribution [1] that is reproduced in any decent text on quantum field theory; see, e.g., $[7,8]$. Insertion of a oneparticle irreducible polarization operator diagram with $n$ photon exchanges in the photon line in Fig. 1 changes the large momentum behavior of the photon propagator by the factor $\sim \ln \left(k_{E}^{2} / \mathrm{m}^{2}\right)$ that does not change the dominant integration momentum region. We see that the characteristic spacelike integration momenta $k^{2}<0$ are separated by a large gap $\sim 4 m^{2}$ from the position of the positronium singularities at timelike momenta $k^{2} \sim 4 m^{2}$. In this kinematics, each polarization operator diagram with an extra Coulomb exchange introduces an extra power of $\alpha$ and to achieve the desired accuracy in calculation of AMM it is sufficient to consider only a finite number of lower-order perturbation theory diagrams. All higher-order diagrams with a larger number of exchanged photons are suppressed by powers of $\alpha$ and can be safely neglected. ${ }^{1}$ The

\footnotetext{
${ }^{1}$ We do not discuss insertions of one-particle reducible polarization operators that lead to the well-known renormalon singularity [9].
}

positronium poles are nothing but the sum of the perturbation theory diagrams at $k^{2} \sim 4 m^{2}$, and we see that for kinematical reasons they do not arise in calculation of the corrections to AMM.

The discussion above can be illustrated with the help of a toy model suggested in [3]. Consider the function $f(x)=$ $a /(x-a)$ (in the context of the present discussion one can think that $x$ is something like $k^{2}-4 m^{2}$, and $a$ is something like $-\alpha^{2} m^{2} / n^{2}$ ). Expanding this function in a power series and removing from this expansion, say, the first ten terms

$$
f(x)=\frac{a}{x}+\frac{a^{2}}{x^{2}}+\cdots+\frac{a^{10}}{x^{10}}+\frac{a^{11}}{x^{11}} \frac{a}{x-a},
$$

we observe that the remainder function $f_{1}(x)=$ $\left(a^{11} / x^{11}\right) a /(x-a)$ still has the pole with the same residue as the original function $f(x)$. This observation was used in [3] as an argument demonstrating that the pole contribution of the function $f_{1}(x)$ has to be accounted for together with the first few terms in the power series expansion. It was claimed that since the residue of the function $f_{1}(x)$ at $x=a$ coincides with the residue of the function $f(x)$, the pole contribution of $f_{1}(x)$ is not suppressed in comparison with the perturbation theory contributions. However, as explained above, we are interested in the value of the integral of this series expansion with a certain weight, and the weight function chooses the values of the argument that are far from the pole. For illustrative purposes, let us choose the weight in the form $\delta(x-b)$, where $|b-a| \gg a$. Then

$f(b)=\int d x f(x) \delta(x-b)=\frac{a}{b}+\frac{a^{2}}{b^{2}}+\cdots+\frac{a^{10}}{b^{10}}+f_{1}(b)$,

where $f_{1}(b)=\left(a^{11} / b^{11}\right)[a /(b-a)]$. We see that the contribution of the "pole" term in $f_{1}(b)$ is purely perturbative in the small parameter $a / b$, and addition to the sum of a few first terms of something like $f(b)=a /(b-a)$ as is effectively done in $[3,4]$ is unjustified. Of course, this toy model is just an illustration of the considerations above.

To summarize, inclusion of the positronium poles (one pole in [3], a series of poles in [4]) on par with a few perturbation theory diagrams is an uncontrollable approximation that leads to wrong results. The positronium poles in the polarization operator arise from summation of the diagrams with any number of the Coulomb exchanges. The poles show up as divergences of this series at specific values of momentum. Necessity for account for the positronium poles arises when all terms in the series are of the same order and the series diverges. In other words the perturbation theory itself prompts when it becomes insufficient. This is not the case for the corrections to AMM, and it makes no sense to add positronium pole terms to the perturbation theory contributions as is done in $[3,4]$. 


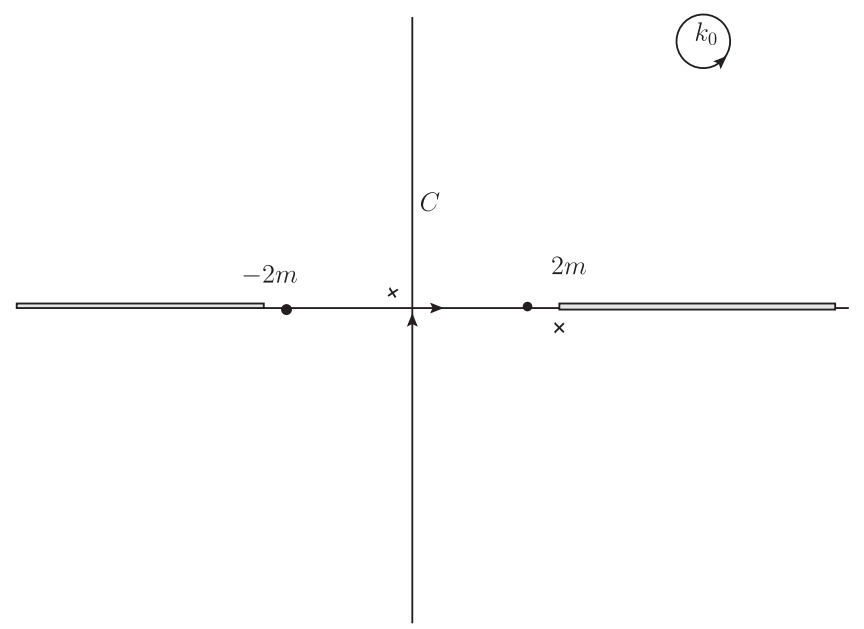

FIG. 2. Singularities in the complex $k_{0}$ plane.

One can prove the purely perturbative nature of the vacuum polarization corrections to AMM from a slightly different perspective [5]. The diagram in Fig. 1 with an exact one-particle irreducible polarization operator contains all polarization operator contributions to AMM. The exact polarization operator has, of course, positronium poles. Let us show that in the diagram in Fig. 1 the contribution of the exact polarization operator reduces to a sum of contributions of a few lower-order perturbation theory diagrams. Consider the loop integration over the loop momentum $k$ that coincides with the photon momentum. The singularities of the integrand in the complex plane $k_{0}$ in Fig. 2 are due to the polarization operator singularities [poles (bold dots) at $k^{2}=E_{n}^{2}=4 m^{2}-\alpha^{2} m^{2} / n^{2}+\alpha^{4} m^{2} /\left(16 n^{2}\right)$ and cuts at $k^{2} \geq 4 m^{2}$. There are also singularities that arise due to other propagators in the triangle diagram (crosses in Fig. 2). We see that, as usual in the diagrammatic calculations, the structure of singularities allows rotation of the integration contour to the imaginary axis. After this rotation, the contour is at a distance about $2 m$ from the positronium singularities. As we discussed above for such momenta, the exact polarization operator is a sum of a convergent perturbation theory expansion in $\alpha$, which proves the validity of perturbation theory for calculation of radiative corrections to AMM.

Notice that in some other cases accounting for the positronium bound states is necessary in QED calculations. This happens when the singularities that depend on external momentum pin the integration contour to the positronium singularity, or when the polarization operator for kinematical reasons should be calculated near the positronium singularities. An example of such situation is provided by the contribution of the one-photon annihilation diagram to the positronium hyperfine splitting. The proper way to treat this problem was discovered and exhaustively discussed in [6].

For completeness let us consider also another argument in favor of accounting for the positronium poles in AMM calculations put forward in [3,4]. It is based on the wellknown dispersion relation representation of the polarization operator contribution to AMM

$$
\Delta a=\frac{\alpha}{\pi^{2}} \int \frac{d s}{s} \operatorname{Im} \Pi(s+i \epsilon) K(s),
$$

where

$$
K(s)=\int_{0}^{1} d x \frac{x^{2}(1-x)}{x^{2}+(1-x) \frac{s}{m^{2}}} .
$$

It is claimed in $[3,4]$ that considering only the perturbative contributions to the imaginary part in Eq. (3) one misses the positronium pole contributions. Let us consider this argument in more detail, and apply it to the dispersion relation for the polarization operator itself. It is clear that each perturbation theory contribution to the polarization operator can be restored from its imaginary part with the help of the dispersion relation. Summing these perturbation theory contributions to the polarization operator, we restore the total polarization operator that contains positronium poles as we explained above. But notice that while restoring this polarization operator via dispersion relations for the perturbation theory diagrams, we never encountered the positronium poles. Is there a contradiction? No, we just observed that the sum of dispersion integrals of perturbative imaginary parts does not coincide with the dispersion integral of the sum of imaginary parts. The summation and integration are in this case noncommutative as was first discovered in [5] (see also [6]). The case of the dispersion integral in Eq. (3) is similar to the case of the dispersion integral for the polarization operator itself, and we should not include positronium singularities in this dispersion relation on par with the perturbation theory contributions for the same reasons. Of course, if we knew the exact expression for the imaginary part of the full polarization operator, we would be able to calculate the total polarization operator contribution to AMM with the help of Eq. (3). Since we do not know the exact polarization operator or its imaginary part, perturbation theory remains the only practical way of calculating the QED corrections to AMM.

In summary, we have considered the arguments in favor of a new nonperturbative correction of order $(\alpha / \pi)^{5}$ to the electron and muon anomalous magnetic moments put forward in $[3,4]$ and proved that such contribution does not exist.

This work was supported by the NSF Grant No. PHY-1066054.

Note added.-Reference [10] appeared on the arXiv on the same day as the present paper and also came to the same conclusions on the anomalous magnetic moment (AMM). The authors of Ref. [4] recently updated their results and acknowledged the absence of nonperturbative QED contributions to lepton AMMs. 


\section{BRIEF REPORTS}

[1] J. Schwinger, Phys. Rev. 73, 416L (1948).

[2] T. Aoyama, M. Hayakawa, T. Kinoshita, and M. Nio, Prog. Theor. Exp. Phys. 2012, 01A107 (2012).

[3] G. Mishima, arXiv:1311.7109.

[4] M. Fael and M. Passera, arXiv:1402.1575 [Phys. Rev. D (to be published)].

[5] M. A. Braun, Zh. Eksp. Teor. Fiz. 54, 1220 (1967) [Sov. Phys. JETP 27, 652 (1968)].

[6] R. Barbieri, P. Christillin, and E. Remiddi, Phys. Rev. A 8, 2266 (1973).
PHYSICAL REVIEW D 90, 057301 (2014)

[7] V. B. Berestetskii, E. M. Lifshitz, and L. P. Pitaevskii, Quantum Electrodynamics, 2nd ed. (Pergamon Press, Oxford, 1982).

[8] M. E. Peskin and D. V. Schroeder, An Introduction to Quantum Field Theory (Addison-Wesley, Reading, Massachusetts, 1995).

[9] B. Lautrup, Phys. Lett. 69B, 109 (1977).

[10] K. Melnikov, A. Vainshtein, and M. Voloshin, Phys. Rev. D 90, 017301 (2014). 